\title{
Efficacy and safety of liposome-paclitaxel/liposome-paclitaxel combined with S-1 in 17 advanced gastric cancer patients with poor performance status
}

\author{
Runhong Wu ${ }^{1 \#}$, Zhu Shen ${ }^{2 \#}, \mathrm{Na} \mathrm{Yu}^{1}$, Jianhao Xu${ }^{1}$, Xuya Yuan ${ }^{1}$, Liwei $\mathrm{Ni}^{1}$, Yuming Long ${ }^{1}$, Jialong Tao ${ }^{1}$, \\ Yusong Zhang ${ }^{1}$ \\ ${ }^{1}$ Department of Oncology, ${ }^{2}$ Department of Pharmacy, the Second Affiliated Hospital of Soochow University, Suzhou 215004, China \\ Contributions: (I) Conception and design: Y Zhang, J Tao; (II) Administrative support: Z Shen; (III) Provision of study materials or patients: R Wu, N \\ Yu; (IV) Collection and assembly of data: R Wu, J Xu, X Yuan, Z Shen, L Ni; (V) Data analysis and interpretation: R Wu, Y long; (VI) Manuscript \\ writing: All authors; (VII) Final approval of manuscript: All authors. \\ \#These authors contributed equally to this work. \\ Correspondence to: Yusong Zhang; Jialong Tao. Department of Oncology, the Second Affiliated Hospital of Soochow University, 1055 Sanxiang Road, \\ Gusu District, Suzhou 215004, China. Email: yusongzhang@126.com; taojialong1221@163.com.
}

Background: To evaluate the efficacy and safety of liposome-paclitaxel (L-PTX)/L-PTX plus S-1 in advanced gastric cancer patients with poor performance status (PS).

Methods: We performed this retrospective study on 17 advanced gastric cancer patients with poor PS [rated as $\geq 2$ based on the Eastern Cooperative Oncology Group (ECOG) scale] who underwent the following chemotherapy regimen: (I) L-PTX single-agent: L-PTX $60-80 \mathrm{mg} / \mathrm{m}^{2}$ given on days 1 and 8 , in a 21-day cycle; (II) timed sequential (TS) regimen: L-PTX $60-80 \mathrm{mg} / \mathrm{m}^{2}$ given on days 1 and 8 . S-1, 40-60 mg/m² twice a day on days 1-14, in a 21-day cycle. Initially, some patients could not tolerate the 2-drug combination chemotherapy regimen, only L-PTX single-agent was given. After the patient's physical condition was improved, plus S-1 was also given.

Results: A total of 17 patients were studied. No complete response (CR) or partial response (PR) were observed in six patients, accounting for $35.29 \%$ (6/17). Stable disease (SD) was observed in five patients, accounting for $29.41 \%$ (5/17), and progressive disease (PD) in 6, accounting for 35.29\% (6/17). The objective response and disease control rates were $35.29 \%(6 / 17)$ and $64.71 \%(11 / 17)$, respectively. The median progression-free survival (PFS) and median overall survival (OS) were 6.50 months [95\% confidence interval (CI): 4.81-8.20] and 13.00 months (95\% CI: $0.00-33.65$ ), respectively. The most common hematological toxicities were neutropenia and anemia.

Conclusions: L-PTX/L-PTX plus S-1 in the treatment of advanced gastric cancer patients with poor PS can prolong the patients' PFS and OS, and the toxicity is tolerable.

Keywords: Liposome-paclitaxel (L-PTX); S-1; advanced gastric cancer; efficacy; safety

Submitted Mar 03, 2019. Accepted for publication Jul 09, 2019.

doi: $10.21037 /$ tcr.2019.08.17

View this article at: http://dx.doi.org/10.21037/tcr.2019.08.17

\section{Introduction}

Gastric cancer is the fifth most common cancer and the third leading cause of cancer deaths worldwide (1). Gastric cancer is one of the most common tumors in China. For patients with gastric cancer, operation is the main treatment to obtain a chance for cure. Chemotherapy is one of the main treatments for prolonging survival and improving quality of life for advanced gastric cancer who are not suitable for surgery or who have undergone postoperative recurrence and metastasis $(2,3)$. However, advanced gastric 
cancer patients with poor performance status (PS) due to multiple organ metastases or celiac metastases, intestinal obstruction, severe anemia, and advanced age are often unable to tolerate high-intensity chemotherapy. The conventional supportive treatment is the best treatment option. The average overall survival (OS) of the best supportive care is about 3 months. For these patients, whether some highly effective and low-toxic chemotherapy regimens may prolong survival and improve quality of life is worthy of clinical exploration.

Paclitaxel is a new anti-microtubule drug that is commonly used for breast cancer, non-small cell lung cancer, gastric cancer, head and neck cancer and other cancers. According to the United States National Comprehensive Cancer Network (NCCN) guidelines (4), taxanes single-agent or in combination with other drugs are recommended as first-line treatment options for advanced gastric cancer. Liposomepaclitaxel (L-PTX) is a liposomal form of paclitaxel with improved efficacy and less side-effects as compared to common paclitaxel. It is considered relatively safe for advanced gastric cancer patients with poor PS.

Phase I clinical studies of liposomal-paclitaxel have been completed. The main pharmacokinetic parameters of L-PTX and conventional paclitaxel (C-PTX) after a single intravenous infusion of $175 \mathrm{mg} / \mathrm{m}^{2}$ were as follows: peak plasma concentrations $\left(\mathrm{C}_{\max }\right)$ were $6,455 \pm 2,247$ and $7,400 \pm 1,542 \mu \mathrm{g} / \mathrm{L}$; the areas under the plasma concentrationtime curve $\left(\mathrm{AUC}_{0-\infty}\right)$ were $14,812 \pm 2,846$ and 21,693 \pm $2,657 \mu \mathrm{g} \cdot \mathrm{h} \cdot \mathrm{L}^{-1}$; the plasma elimination half-life $\left(\mathrm{t}_{1 / 2 z}\right)$ were $30.5 \pm 7.3$ and $13.7 \pm 3.2 \mathrm{~h}$; the apparent volumes of distribution $\left(\mathrm{V}_{\mathrm{z}}\right)$ were $526.8 \pm 112.1$ and $162.9 \pm 49.1 \mathrm{~L} / \mathrm{m}^{2}$; the plasma clearance rates $\left(\mathrm{CL}_{z}\right)$ were $12.3 \pm 2.7$ and $8.2 \pm 1.0 \mathrm{~L} \cdot \mathrm{h}^{-1} \cdot \mathrm{m}^{-2}$, respectively. The statistical analysis showed that there was a significant difference in major pharmacokinetic parameters between L-PTX and C-PTX $(\mathrm{P}<0.05)(5)$.

Fluorouracil is widely used in the treatment of gastric cancer. S-1, an orally administered fluorouracil, is used clinically to treat gastric and head and neck cancers. It is a compound containing tegafur, 5-chloro-2,4dihydroxypyridine, and potassium oxonate with a molar ratio of 1:0.4:1 $(6,7)$. Some studies have shown that $\mathrm{S}-1$ has lower gastrointestinal toxicity than $5-\mathrm{Fu}$, but their antitumor effects are not significantly higher (5). S-1 has good efficacy in the treatment of advanced gastric cancer. The combination of S-1 and other chemotherapeutic agents can achieve better chemotherapeutic effect than S-1 alone (8). A multicenter phase II clinical trial showed that weekly treatment with paclitaxel and S-1 in advanced gastric cancer can achieve a considerable survival time and low toxicity (9).

L-PTX has fewer adverse reactions than paclitaxel in patients with advanced gastric cancer (10). The short-term efficacy of L-PTX plus S-1 compared with oxaliplatin plus S-1 was similar, but the L-PTX group had lower toxicity and could be used to treat elderly patients with advanced gastric cancer (11). Therefore, we used L-PTX single-agent or L-PTX plus S-1 to treat advanced gastric cancer patients PS scores $\geq 2$.

\section{Methods}

\section{Patients selection}

This study included a retrospective collection of 17 advanced gastric cancer patients with poor PS who were treated in our hospital from July 2015 to March 2018. The diagnosis of gastric cancer was confirmed by endoscopic biopsy or postoperative pathology. Gastric cancer was in an advanced stage or had recurred and metastasized at the time of diagnosis. The patients are required to meet at least one of the following conditions: (I) patients older than 70 years, (II) patients having intestinal obstruction or celiac metastasis, and (III) patients with multiple organ metastases. All patients had poor PS due to the above characteristics. Their PS was rated as $\geq 2$ according to the Eastern Cooperative Oncology Group (ECOG) scale, and the patients could not tolerate routine high-intensity chemotherapy. This study was approved by the Research Ethics Committee at the Second Affiliated Hospital of Soochow University. All patients signed the informed consent before enrolling into this study and also released the use of their clinical data in this study.

\section{Treatment methods}

L-PTX single-agent referred to $60-80 \mathrm{mg} / \mathrm{m}^{2}$ given on days 1 and 8 , in a 21-day cycle. Some patients were in extremely poor condition, the total amount of L-PTX was divided into three doses (days 1, 8, and 15). If the general condition of the patient was extremely poor, the total amount of L-PTX was divided into three doses (days 1, 8, and 15). Timed sequential (TS) regimen: L-PTX $60-80 \mathrm{mg} / \mathrm{m}^{2}$ given on days 1 and 8 . S-1, 40-60 mg/m $\mathrm{m}^{2}$ twice a day on days $1-14$, in a 21-day cycle. The dose of L-PTX or S-1 was based on the patient's body surface area and PS. The course of treatment was 3 weeks and continued until six sessions or patient's disease progression, intolerable toxicity, and death. Disease 
progression was indicated by computed tomography (CT) showing disease progression, significant increase in ascites, significant increase in intestinal obstruction. Toxic reactions that were difficult to tolerate include hematological toxicity (grade 4 leukopenia or neutropenia, grade 3 febrile neutropenia, grade 3 thrombocytopenia). Some patients initially could not tolerate the 2-drug combination chemotherapy regimen, only L-PTX single-agent chemotherapy was given or low-dose chemotherapy was given first. After the patient's physical condition improves, plus S-1 was also given other dose of L-PTX was increased.

\section{Efficacy and toxicity evaluation}

The patients were followed up every 3 months until the patient died or March 2018, whichever came first. All patients were evaluated for tumor response, including complete response (CR), partial response (PR), stable disease (SD), and progressive disease (PD), according to the response evaluation criteria in solid tumors guidelines (version 1.1). The evaluation criteria for the remission of lesions that could not be assessed by imaging were: (I) Significant reduction in ascites; (II) improvement in digestive tract obstruction; (III) anemia improved. Adverse events were graded according to the National Cancer Institute (NCI) common toxicity criteria (CIC) (version 4.0). Detection of adverse events continues throughout the course of treatment.

\section{Result analysis}

Progression-free survival (PFS) was recorded as the time from the date of entry into the study to the time of disease progression or death without evidence of progression occurred. OS was recorded from the time of diagnosed with gastric cancer to the time of death or last follow-up visit. The objective response rate (ORR) was the sum of CR and $\mathrm{PR}$, and the disease control rate was the sum of CR, PR, and SD. Median OS and PFS were calculated using the Kaplan-Meier method.

\section{Results}

\section{Patients characteristics}

From July 2015 to March 2018, we observed a total of 17 patients. The median age was 64 years (age distribution: 23-81 years; 7 females and 10 males). The pathological types were poorly differentiated adenocarcinoma in 8 cases and adenocarcinoma in 7 cases. Four patients were older than 75 years old, 3 patients had intestinal obstruction, and 12 had celiac metastases.

Ten patients had an advanced stage of cancer at the time of diagnosis and were unable to undergo surgery. Four patients had undergone radical surgery. One patient had undergone adjuvant chemotherapy with oxaliplatin + S-1 (SOX) after surgery, two patients had received adjuvant chemotherapy with oxaliplatin + capecitabine (XELOX), and one patient had received adjuvant chemotherapy with oxaliplatin + raltitrexed. All patients had a PS score $\geq 2$.

Of the 17 patients, 15 were advanced first-line chemotherapy. Three patients had recurrence after radical gastrectomy. Two of the 17 patients were advanced secondline chemotherapy. Three patients were given L-PTX monotherapy first, and after general improvement, they were given L-PTX + S-1. The remaining patients were treated with L-PTX + S-1 chemotherapy directly.

Among the 17 patients, four were given irinotecan, leucovorin and 5-FU (FOLFIRI) regimen as second-line chemotherapy after disease progression, two of whom also received cisplatin intraperitoneal chemotherapy (Table 1).

\section{ORR and survival time}

In all patients, there was no CR. PR was observed in 6 cases, accounting for $35.29 \%$ (6/17); SD in 5 cases, accounting for $29.41 \%(5 / 17)$; and PD in 6 cases, accounting for $35.29 \%$ (6/17). The ORR and disease control rate were $35.29 \%$ $(6 / 17)$ and $64.71 \%(11 / 17)$, respectively (Table 2). The median PFS was 6.50 months [95\% confidence interval (CI): 4.81-8.20], and the median OS was 13.00 months (95\% CI: 0.00-33.65) (Figure 1A,B). The median PFS for PS groups 2, 3 and 4 was 4, 5.75 and 6.5 months, respectively. The median OS for PS groups 2, 3 and 4 was 9, 8.5 and 29 months, respectively. The PFS rates in 3, 6 , and 9 months were $76.47 \%$ (13/17), $47.06 \%$ (8/17), and $29.41 \%(5 / 17)$, respectively. The OS rates in 3, 6, and 9 months were $100 \%$ (17/17), 82.35\% (14/17), and 70.59\% (12/17), respectively.

\section{Typical case 1}

The patient was a 50-year-old man. He received XELOX (capecitabine and oxaliplatin) regimen for half a year after radical total gastrectomy. Approximately 1.5 years after the end of chemotherapy, the patient developed celiac metastasis with intestinal obstruction (Figure $2 A$ ) and hypokalemia. 
Table 1 Summary of patient characteristics

\begin{tabular}{|c|c|}
\hline Included patients & $\mathrm{n}=17(\%)$ \\
\hline \multicolumn{2}{|l|}{ Age (years) } \\
\hline Median (min-max) & 64 [23-81] \\
\hline \multicolumn{2}{|l|}{ Sex } \\
\hline Male & $10(58.82)$ \\
\hline Female & $7(41.18)$ \\
\hline \multicolumn{2}{|l|}{ ECOG } \\
\hline 2 & $3(17.65)$ \\
\hline 3 & $10(58.82)$ \\
\hline 4 & $4(23.53)$ \\
\hline \multicolumn{2}{|l|}{ Histology } \\
\hline Low-middle differentiated & $1(5.82)$ \\
\hline Poorly differentiated & $7(41.18)$ \\
\hline Adenocarcinoma & $7(41.18)$ \\
\hline Unknown & $1(5.82)$ \\
\hline \multicolumn{2}{|l|}{ Surgery } \\
\hline \multicolumn{2}{|l|}{ Yes } \\
\hline Radical & $4(23.53)$ \\
\hline Palliative & $3(17.65)$ \\
\hline No & $10(58.82)$ \\
\hline \multicolumn{2}{|l|}{ Patients' condition } \\
\hline Digestive tract obstruction & 8 \\
\hline Age greater than 75 years old & 4 \\
\hline Moderate to severe anemia & 6 \\
\hline \multicolumn{2}{|l|}{ Metastasis sites } \\
\hline Liver & 4 \\
\hline Lymph node & 4 \\
\hline Peritoneal & 13 \\
\hline Lung & 1 \\
\hline Bone & 1 \\
\hline Total & 23 \\
\hline \multicolumn{2}{|l|}{ Second line } \\
\hline FOLFIRI & 4 \\
\hline Cisplatin intraperitoneal & 2 \\
\hline Best supportive care & 11 \\
\hline
\end{tabular}

Table 2 Summary of recent efficacy $(\mathrm{n}=17)$

\begin{tabular}{lc}
\hline Recent efficacy & Patient number \\
\hline CR & 0 \\
PR & 6 \\
SD & 5 \\
PD & 6 \\
ORR (\%) & $6 / 17(35.29 \%)$ \\
Disease control rate (\%) & $11 / 17(64.71 \%)$ \\
\hline
\end{tabular}

$\mathrm{CR}$, complete response; PR, partial response; SD, stable disease; PD, progressive disease; ORR, objective response rate.
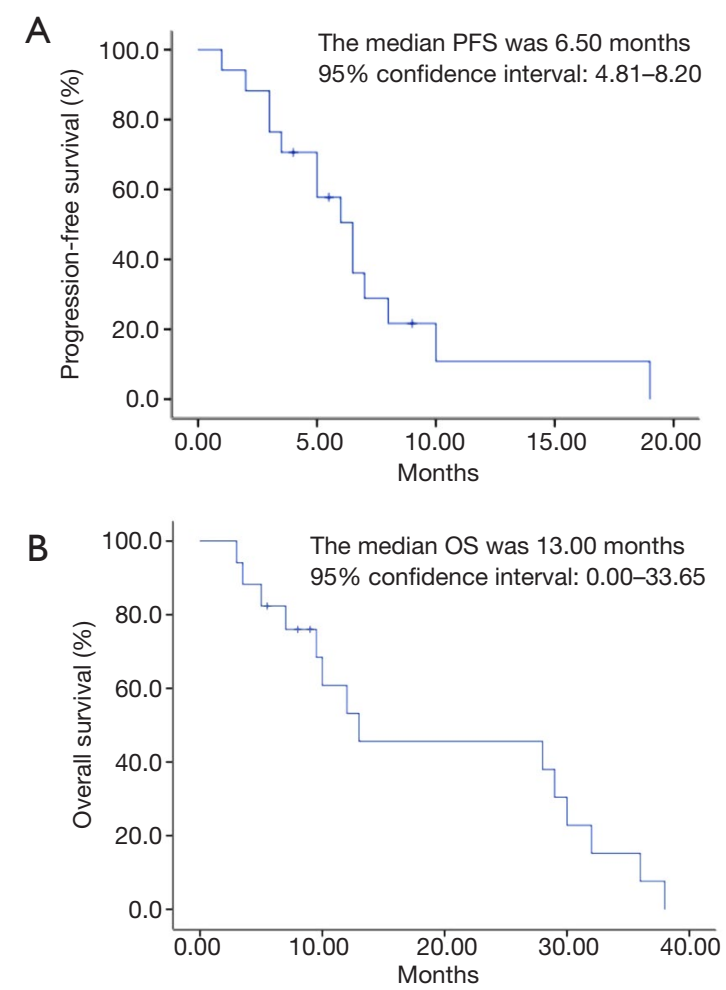

Figure 1 Survival analysis of PFS (A) and OS (B). PFS, progressionfree survival; OS, overall survival.

After the best supportive care, the patient was given L-PTX single-drug (60 mg d1 + $90 \mathrm{mg} \mathrm{d} 8,15)$ chemotherapy. After one cycle of chemotherapy, the patient's bowel movements gradually became unobstructed. He was then given four regimens of TS (L-PTX $120 \mathrm{mg}$ given on days 1 and 8 combined with S-1 $80 \mathrm{mg}$ twice a day on days 1-14, 3 weeks as one cycle). After 4 times of TS regimens, the 

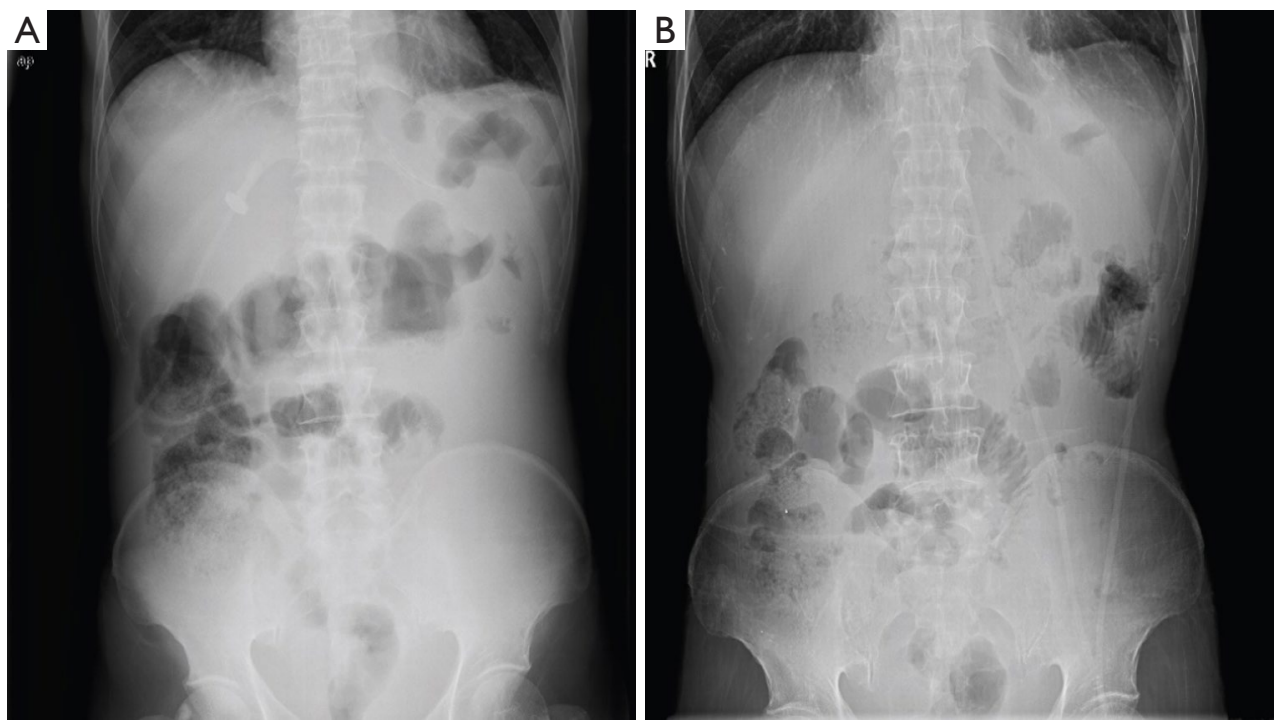

Figure 2 Representative CT abdominal plain images of a patient before (A) and after (B) the application of TS regimens. The patient developed intestinal obstruction due to abdominal metastasis 1.5 years after the end of initial chemotherapy course. After 4 times of TS regimens, the intestinal obstruction was disappeared. CT, computed tomography; TS, timed sequential.

intestinal obstruction was eliminated (Figure $2 B$ ). The patient's blood potassium level returned to normal, and weight increased by $20 \mathrm{~kg}$.

\section{Typical case 2}

The patient was a 56-year-old man who had stomach and cardiac cancer involving the lower esophagus. $\mathrm{He}$ underwent an operation in the lower esophagus due to eating obstruction. Postoperatively, he was given six regimens of chemotherapy with "docetaxel + cisplatin" and was maintained on docetaxel single-agent 2 times. However, several changes were made in the chemotherapy regimen due to disease progression. He had an esophageal stenosis in December 2015 (Figure 3A). After receiving L-PTX single-agent treatment (L-PTX $120 \mathrm{mg}$ given on days 1 and 8), his esophageal stenosis was significantly improved (Figure 3B). Afterward, he was given five regimens of TS (L-PTX $120 \mathrm{mg}$ given on days 1 and 8 combined with S-1 $120 \mathrm{mg}$ twice a day on days $1-14,3$ weeks as one cycle).

\section{Toxicity}

Hematological and non-hematological toxicities are listed in Table 3. The most common hematological toxicities were neutropenia and anemia with incidence rates of $35.29 \%$
$(6 / 17)$ and $88.24 \%(15 / 17)$, respectively. One patient $(5.88 \%)$ had grade 2 neutropenia, and no patient had grade 3 or grade 4 neutropenia. Five patients $(29.41 \%)$ had grade 3 anemia. Non-hematologic toxicity was mild. Nausea accounted for $41.18 \%$ (7/17), and other non-hematologic toxicities included fatigue $(4 / 17,23.53 \%)$, vomiting $(7 / 17$, $41.48 \%)$, elevated transaminases $(1 / 17,5.88 \%)$, and diarrhea $(5 / 17,29.41 \%)$. No patient experienced grade 4 non-hematologic toxicity. These toxic reactions improved after symptomatic treatment, and no treatment-related deaths occurred.

\section{Discussion}

The incidence of gastric cancer is much higher in China compared with other countries. In China, due to economic conditions and inadequate attention to the disease, some patients are already in advanced stages at the time of initial diagnosis. Several studies suggest that systemic chemotherapy is superior to best supportive care for advanced gastric cancer (12-14). Compared with best supportive care alone, chemotherapy plus best supportive care can extend OS of advanced gastric cancer by 2 months (12). A recent meta-analysis showed a median OS of 3.2 months for best supportive care in advanced gastric cancer patients with PS scores of $0-1$ points (15). The US 

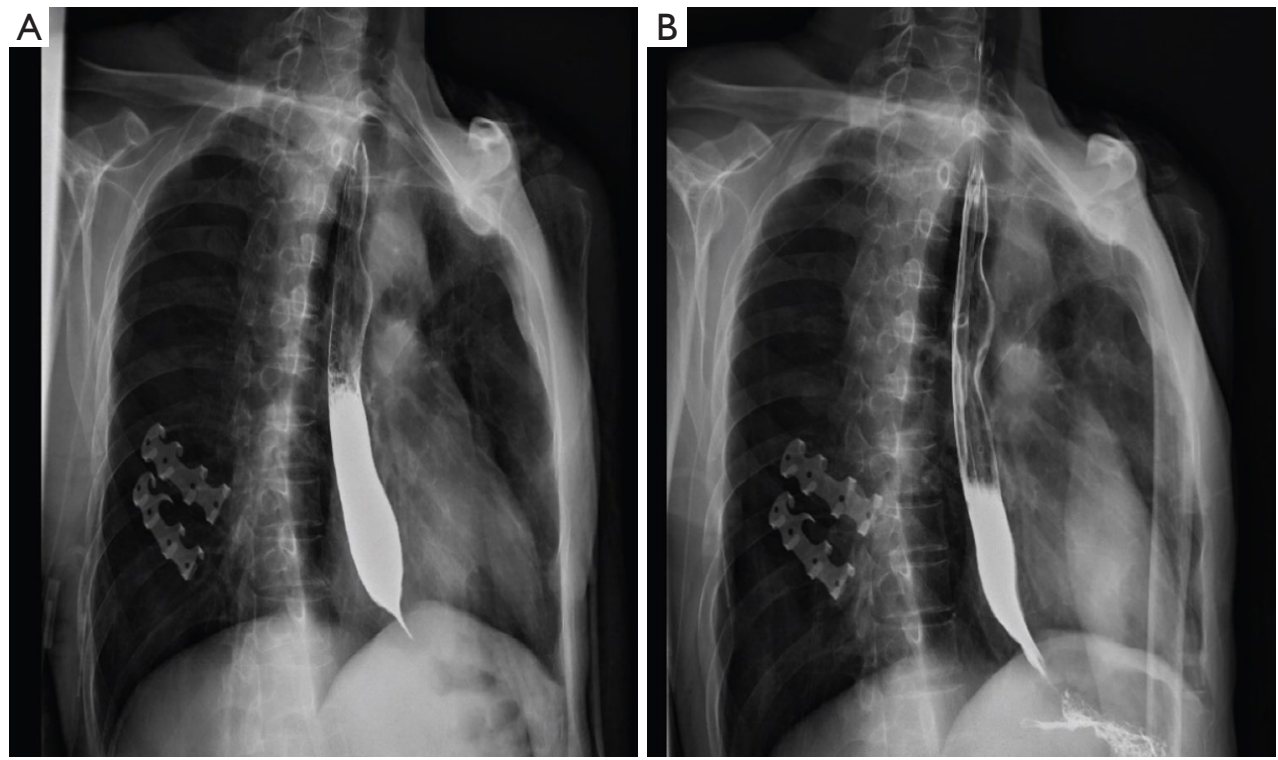

Figure 3 Representative CT chest plain images of a patient before (A) and after (B) the application of TS regimens. The patient had an esophageal stenosis, which was significantly improved after receiving one time of L-PTX single-agent treatment. CT, computed tomography; TS, timed sequential; L-PTX, liposome-paclitaxel.

Table 3 Summary of treatment toxicity

\begin{tabular}{lcc}
\hline Toxicity NCI CTC term & $\begin{array}{c}\text { All grades, } \\
\mathrm{n}(\%)\end{array}$ & $\begin{array}{c}\text { Grade 3-4, } \\
\mathrm{n}(\%)\end{array}$ \\
\hline $\begin{array}{l}\text { Hematological } \\
\text { Neutropenia }\end{array}$ & $\begin{array}{c}6(35.29) \\
\text { Anemia }\end{array}$ & $0(0.00)$ \\
Non-hematological & & $5(29.41)$ \\
Nausea & $7(41.18)$ & $0(0.00)$ \\
Fatigue & $4(23.53)$ & $0(0.00)$ \\
Vomiting & $7(41.48)$ & $0(0.00)$ \\
Transaminases increased & $1(5.88)$ & $0(0.00)$ \\
Diarrhea & $5(29.41)$ & $0(0.00)$ \\
All & 45 & 5 \\
\hline
\end{tabular}

$\mathrm{NCl}$, National Cancer Institute; CTC, common toxicity criteria.

NCCN guidelines (4) recommend chemotherapy with best supportive care for advanced gastric cancer patients with a PS score of $\leq 2$ and best supportive care for those with a PS score of $>2$. However, the guidelines also indicate that the recommendations are applicable to the general status of most patients and may not be applicable to all patients (4). Specifically, each patient should be given individualized treatment options according to specific conditions. Our clinical experience shows that not all patients with PS scores $\geq 2$ can receive active antitumor therapy. In gastric cancer patients with a PS score $\geq 2$, poor PS usually results from two causes. One is that there are other diseases combined with gastric cancer, and the other is due to digestive tract obstruction and anemia caused by the tumor itself. In the latter case, if the tumor load can be reduced by active antitumor therapy, the patient's physical condition can be improved, and he/she can receive follow-up treatment, providing an opportunity to extend the life span and improve the quality of life. However, these patients have difficulty tolerating conventional regimens and doses of chemotherapy; therefore, a highly effective and low-toxicity chemotherapy regimen must be explored.

Current chemotherapy drugs used for the treatment of advanced gastric cancer include taxanes, fluorouracils (5-Fu, S-1, capecitabine), irinotecan, platinum drugs (cisplatin, carboplatin, oxaliplatin), etc. However, there is no standard advanced first-line chemotherapy regimen (16). Multiple phase I and phase II clinical trials have shown that weekly treatment of advanced gastric cancer with paclitaxel combined with S-1 demonstrated tolerable toxicity and efficacy (17-19). A phase II multicenter prospective randomized clinical trial concluded that S-1 plus paclitaxel and S-1 plus cisplatin are both effective 
in patients with advanced gastric cancer (20). However, S-1 plus paclitaxel has better efficacy and tolerability in gastric cancer patients with abdominal metastasis (21). The patients we have observed for gastric cancer, the poor PS of the patients was due to tumors rather than comorbidities. The chemotherapy regimen used was L-PTX or L-PTX plus S-1 chemotherapy. L-PTX is a paclitaxel liposome that was successfully developed in China in 2003. The use of liposomes instead of polyoxyethylene castor oil and absolute ethanol compound media can reduce paclitaxel toxicity and solvent-induced allergic reactions $(22,23)$. Clinical pharmacokinetic tests showed that the elimination half-life of paclitaxel liposomes is significantly longer than that of paclitaxel injection, which allows tumor cells to be exposed to an effective killing concentration of paclitaxel for a longer period of time. In addition, the liposome preparation has a passive targeting property, which is better than that of paclitaxel in tumor tissues and is significantly poorer than that of C-PTX in normal tissue and blood (24). Therefore, liposomal-paclitaxel has advantages over traditional paclitaxel in enhancing drug targeting, reducing adverse drug reactions, and improving body tolerance. In our previous study on the use of liposomal-paclitaxel in advanced gastric cancer patients with better physical status, we found that liposomal-paclitaxel has a comparable efficacy to that of paclitaxel, and it is well tolerated. Therefore, we observed the efficacy and safety of L-PTX/L-PTX combined with S-1 in advanced gastric cancer patients with poor PS. The results showed that the chemotherapy regimen can prolong the survival of the patients and improve their quality of life.

Of the 17 patients, five had cardia or pyloric obstruction due to tumors, and three developed intestinal obstruction due to peritoneal metastasis. As these patients are unable to eat, they are generally in poor PS. On the basis of active parenteral nutrition, L-PTX single-agent was given. After 1-2 cycles of treatment, the obstruction in the digestive tract gradually disappeared, and the patients' diet gradually returned to normal. Then with or without S-1, the patients' survival time was extended, and their quality of life improved. This finding shows that for advanced gastric cancer patients with tumor-induced digestive tract obstruction, L-PTX single-drug chemotherapy can be given on the basis of adequate parenteral nutrition. If the treatment is effective, patients can receive further treatment, and their quality of life can be improved, extending their survival time.

Among the patients that we treated, six had poor physical condition due to moderate to severe anemia caused by tumor hemorrhage. For patients with hemoglobin below $60 \mathrm{~g} / \mathrm{L}$, chemotherapy is given concurrently with transfusion therapy. In the treatment of patients with hemoglobin of more than $60 \mathrm{~g} / \mathrm{L}$, because of non-compliance with Chinese blood transfusion regulations, the above regimen will be given on the basis of adequate parenteral nutrition support treatment. The results showed that after treatment, four patients had reduced tumor burden and hemorrhage, gradually increased hemoglobin, and improved physical status. These patients also had the opportunity to continue follow-up treatment, improve their quality of life, and prolong survival.

In the treatment of malignant tumors, age is also a factor to be considered. Among the 17 patients, four were older than 75 years. Combined with digestive tract obstruction or anemia, these patients had poor PS, but we still administered liposomal-paclitaxel or liposomal-paclitaxel combined with S-1. The results showed that an 80-year-old patient had obstruction due to stomach cancer, and her obstruction disappeared after one cycle of L-PTX singleagent chemotherapy. After the administration of liposomalpaclitaxel combined with S-1 for six cycles, the patient's OS reached 28 months.

Besides the therapeutic effect, we also observed the side effects of treatment. The results showed that the side effects of liposomal paclitaxel single-agent or liposomal paclitaxel plus S-1 were mild, and the common toxicity was 1-2 grade neutropenia and anemia. And patients can be well tolerated after symptomatic and supportive treatment. Therefore, advanced gastric cancer patients with a PS score $\geq 2$ should be distinguished. If the patient's poor physical status is caused by the tumor itself, systemic chemotherapy can be given on the basis of adequate supportive care. The choice of chemotherapy regimen and chemotherapy drug needs to be individualized according to the patient's physical status. Because L-PTX has high efficacy and low toxicity, it can be considered for advanced gastric cancer with poor physical status, especially those with digestive tract obstruction and who are unable to eat. When the treatment is effective and the obstruction is relieved, $\mathrm{S} 1$ can be added to increase the therapeutic effect. Patients who do not have digestive tract obstruction and are able to eat can be directly given a combination chemotherapy based on his/her physical status to obtain rapid improvement of his/her condition.

Because the proportion of advanced gastric cancer patients with PS score $\geq 2$ is very low among patients with gastric cancer, the proportion of patients with poor physical 
status caused by the tumor itself is even smaller. As of now, we only observed 17 patients. There are still several deficiencies in the data, and a large phase III clinical trial is needed to verify our results.

In summary, if the poor physical status of advanced gastric cancer patients is caused by tumor-induced digestive tract obstruction, anemia, etc., systemic chemotherapy can be considered. The chemotherapy program requires drugs with high efficiency and low toxicity. Our results show that L-PTX single-agent or L-PTX plus S-1 has a potential to be used for the treatment of advanced gastric cancer patients.

\section{Acknowledgments}

Funding: The current study was supported by grants from Bureau of Public Health of Jiangsu Province (H201413), the second affiliated hospital of Soochow university pre-research project (SDFEYGJ1609), the second affiliated hospital of Soochow university clinical discipline group project funding (XKQ 2015008) and the project from national key laboratory of radiation medicine (GZK1201820).

\section{Footnote}

Conflict of Interest: All authors have completed the ICMJE uniform disclosure form (available at http://dx.doi. org/10.21037/tcr.2019.08.17). The authors have no conflicts of interest to declare.

Ethical Statement: The authors are accountable for all aspects of the work in ensuring that questions related to the accuracy or integrity of any part of the work are appropriately investigated and resolved. The study was conducted in accordance with the Declaration of Helsinki (as revised in 2013). This study was approved by the Research Ethics Committee at the Second Affiliated Hospital of Soochow University (JD-LK-2019-089-01). All patients signed the informed consent before enrolling into this study and also released the use of their clinical data in this study.

Open Access Statement: This is an Open Access article distributed in accordance with the Creative Commons Attribution-NonCommercial-NoDerivs 4.0 International License (CC BY-NC-ND 4.0), which permits the noncommercial replication and distribution of the article with the strict proviso that no changes or edits are made and the original work is properly cited (including links to both the formal publication through the relevant DOI and the license). See: https://creativecommons.org/licenses/by-nc-nd/4.0/.

\section{References}

1. Bray F, Ferlay J, Soerjomataram I, et al. Global cancer statistics 2018: GLOBOCAN estimates of incidence and mortality worldwide for 36 cancers in 185 countries. CA Cancer J Clin 2018;68:394-424.

2 Pyrhönen S, Kuitunen T, Nyandoto P, et al. Randomised comparison of fluorouracil, epidoxorubicin and methotrexate (FEMTX) plus supportive care with supportive care alone in patients with non-resectable gastric cancer. Br J Cancer 1995;71:587-91.

3 Glimelius B, Ekström K, Hoffman K, et al. Randomized comparison between chemotherapy plus best supportive care with best supportive care in advanced gastric cancer. Ann Oncol 1997;8:163-8.

4 Ajani JA, D'Amico TA, Almhanna K, et al. Gastric cancer, version 3.2016, NCCN clinical practice guidelines in oncology. J Natl Compr Canc Netw 2016;14:1286-312.

5 Qian J, Wang Y, Yu Y, et al. A comparison of pharmacokinetics between paclitaxel liposome for injection and commercial paclitaxel injection in patients with cancer. Tumor 2011;31:1103-7.

6 Fukushima M, Satake H, Uchida J, et al. Preclinical antitumor efficacy of S-1: a new oral formulation of 5 -fluorouracil on human tumor xenografts. Int J Oncol 1998;13:693-8.

7 Sakata Y, Ohtsu A, Horikoshi N, et al. Late phase II study of novel oral fluoropyrimidine anticancer drug S-1 (1 M tegafur- $0.4 \mathrm{M}$ gimestat- $1 \mathrm{M}$ otastat potassium) in advanced gastric cancer patients. Eur J Cancer 1998;34:1715-20.

8 Koizumi W, Kurihara M, Nakano S, et al. Phase II study of S-1, a novel oral derivative of 5 -fluorouracil, in advanced gastric cancer. For the S-1 cooperative gastric cancer study group. Oncology 2000;58:191-7.

9 Ueda Y, Yamagishi H, Ichikawa D, et al. Multicenter phase II study of weekly paclitaxel plus S-1 combination chemotherapy in patients with advanced gastric cancer. Gastric Cancer 2010;13:149-54.

10 Zhang H, Shi J, Zhu F. Effect of paclitaxel liposomes in patients with advanced gastric cancer and various tumor markers. Oncology Progress 2018;16:1261-71.

11 Shen X, Zhuang Z, Gan L, et al. Efficacy and safety of weekly liposome-paclitaxel or oxaliplatin combined with tegafur on elderly advanced gastric cancer. Cancer Res Prev Treat 2016;43:400-3. 
12 Janowitz T, Thuss-Patience P, Marshall A, et al. Chemotherapy vs supportive care alone for relapsed gastric, gastroesophageal junction, and oesophageal adenocarcinoma: a meta-analysis of patient-level data. Br J Cancer 2016;114:381-7.

13 Kim HS, Kim HJ, Kim SY, et al. Second-line chemotherapy versus supportive cancer treatment in advanced gastric cancer: a meta-analysis. Ann Oncol 2013;24:2850-4.

14 Kang JH, Lee SI, Lim DH, et al. Salvage chemotherapy for pretreated gastric cancer: a randomized phase III trial comparing chemotherapy plus best supportive care with best supportive care alone. J Clin Oncol 2012;30:1513-8.

15 Chan WL, Yuen KK, Siu SW, et al. Third-line systemic treatment versus best supportive care for advanced/ metastatic gastric cancer: a systematic review and metaanalysis. Crit Rev Oncol Hematol 2017;116:68-81.

16 Murad AM, Santiago FF, Petroianu A, et al. Modified therapy with 5-fluorouracil, doxorubicin, and methotrexate in advanced gastric cancer. Cancer 1993;72:37-41.

17 Narahara H, Fujitani K, Takiuchi H, et al. Phase II study of a combination of S-1 and paclitaxel in patients with unresectable or metastatic gastric cancer. Oncology 2008;74:37-41.

18 Kawabata R, Fujiwara Y, Doki Y, et al. Phase I/II study of a combination of S-1 and weekly paclitaxel in patients with advanced or recurrent gastric cancer. Oncology

Cite this article as: $\mathrm{Wu} \mathrm{R}$, Shen $\mathrm{Z}, \mathrm{Yu} \mathrm{N}, \mathrm{Xu} \mathrm{J}$, Yuan $\mathrm{X}, \mathrm{Ni}$ L, Long Y, Tao J, Zhang Y. Efficacy and safety of liposomepaclitaxel/liposome-paclitaxel combined with S-1 in 17 advanced gastric cancer patients with poor performance status. Transl Cancer Res 2019;8(5):1690-1698. doi: 10.21037/ tcr.2019.08.17
2007;72:219-25.

19 Inada S, Tomidokoro T, Fukunari H, et al. Phase I/II trial of combination therapy with S-1 and weekly paclitaxel in patients with unresectable or recurrent gastric cancer. Cancer Chemother Pharmacol 2009;63:267-73.

20 Mochiki E, Ogata K, Ohno T, et al. Phase II multiinstitutional prospective randomised trial comparing S-1+paclitaxel with $\mathrm{S}-1+$ cisplatin in patients with unresectable and/or recurrent advanced gastric cancer. $\mathrm{Br} \mathrm{J}$ Cancer 2012;107:31-6.

21 Ishigami H, Kitayama J, Kaisaki S, et al. Phase II study of weekly intravenous and intraperitoneal paclitaxel combined with S-1 for advanced gastric cancer with peritoneal metastasis. Ann Oncol 2010;21:67-70.

22 Zhang W, Shi Y, Chen Y, et al. Enhanced antitumor efficacy by paclitaxel-loaded pluronic P123/F127 mixed micelles against non-small cell lung cancer based on passive tumor targeting and modulation of drug resistance. Eur J Pharm Biopharm 2010;75:341-53.

23 Sharma D, Chelvi TP, Kaur J, et al. Novel Taxol formulation: polyvinylpyrrolidone nanoparticleencapsulated Taxol for drug delivery in cancer therapy. Oncol Res 1996;8:281-6.

24 Yoshizawa Y, Kono Y, Ogawara K, et al. PEG liposomalization of paclitaxel improved its in vivo disposition and anti-tumor efficacy. Int J Pharm 2011;412:132-41. 